\title{
A Case of an Extremely Low Birth Weight Infant with Morganella morganii Bacteremia and Peritonitis
}

\author{
Betty Pham, MD ${ }^{1}$ Anne Denslow, PA ${ }^{2}$ Michel Mikhael, MD ${ }^{2}$ Jina Lim, MD \\ ${ }^{1}$ Department of Pediatrics, University of California Irvine, Irvine, \\ California \\ 2 Neonatal-Perinatal Medicine Division, Children's Hospital of Orange \\ County, Orange, California \\ Address for correspondence Jina Lim, MD, Neonatal-Perinatal \\ Medicine Division, Children's Hospital of Orange County, 1201 W La \\ Veta Ave, Orange, CA 92868 (e-mail: jlim@choc.org).
}

AJP Rep 2021;11:e113-e118.

\begin{abstract}
Keywords

- Morganella morganii

- spontaneous intestinal perforation

- neonatal sepsis

We describe a case of late onset Morganella morganii sepsis in an extremely low birth weight male neonate born at 23 and $4 / 7$ weeks gestational age to a 30-year-old primigravid mother due to preterm labor. The mother was otherwise healthy with an unremarkable prenatal course. She received steroids and ampicillin prior to delivery. While initial blood cultures were negative, at day of life 4, the neonate developed signs of sepsis with leukocytosis and bandemia, and subsequent blood culture demonstrated growth of M. morganii. The patient then had spontaneous intestinal perforation on day of life 8 with peritoneal cultures growing $M$. morganii. The infant responded to standard therapy and survived to discharge, with few mild developmental delays upon outpatient follow-up. While M. morganii has been demonstrated in the neonatal population, it generally causes early onset sepsis and is associated with high mortality in preterm neonates. Here, we present this case of late onset neonatal sepsis with $M$. morganii complicated by spontaneous intestinal perforation, with survival in a 23 weeks gestation infant.
\end{abstract}

Sepsis is a cause of mortality and morbidity in the neonatal population, with the incidence in the United States of one to four in 1,000 live births. Neonatal sepsis is categorized by the timing of onset, with early onset sepsis (EOS) occurring within 72 hours of life and late onset sepsis (LOS) greater than 72 hours of life. EOS generally represents vertical mother-to-infant transmission, while LOS represents organisms acquired from the hospital environment or community.

EOS typically occurs with Group B streptococcus (GBS), Escherichia coli (E. coli), and Listeria monocytogenes. ${ }^{2}$ Of these organisms, GBS and E. coli account for $75 \%$ of EOS cases ${ }^{3}$ with $E$. coli seen more frequently in preterm neonates. ${ }^{2}$ LOS is most likely due to gram-positive organisms, with

received

April 3, 2021

accepted after revision

June 7,2021 coagulase-negative Staphylococcus being attributed to $70 \%$ of infections. ${ }^{4}$

An uncommon gram-negative bacterium, Morganella morganii (M. morganii), has been identified as a rare cause of adult sepsis, with one study demonstrating Morganella in $1.47 \%$ of gram-negative sepsis ${ }^{5}$ and bacteremia in 10 to $25 \%$ of cases of Morganella infections. ${ }^{6}$ M. morganii is a facultative, anaerobic, gram-negative rod, initially isolated from a pediatric stool culture in 1906, and found commonly in nosocomial infections of the gastrointestinal (GI) and genitourinary tracts of adults. ${ }^{5}$ In the neonatal population, M. morganii has been identified as a rare cause of neonatal sepsis. In a small case review series, M. morganii sepsis appears to be associated with EOS and higher mortality
(C) 2021. The Author(s).

This is an open access article published by Thieme under the terms of the Creative Commons Attribution-NonDerivative-NonCommercial-License, permitting copying and reproduction so long as the original work is given appropriate credit. Contents may not be used for commercial purposes, or adapted, remixed, transformed or built upon. (https://creativecommons.org/ licenses/by-nc-nd/4.0/)

Thieme Medical Publishers, Inc., 333 Seventh Avenue, 18th Floor, New York, NY 10001, USA 
and morbidity in premature and low birth weight (BW) infants. ${ }^{7}$ Few cases of LOS with M. morganii have been reported. ${ }^{3,8,9}$ We report a case of an extremely premature neonate with late onset $M$. morganii sepsis and peritonitis who survived and provide a literature review of $M$. morganii sepsis.

\section{Case Report}

A 30 -year-old gravida 1, para 0 mother presented and was admitted to a local hospital at 21 weeks gestation for preterm labor. Her prenatal laboratories were unremarkable, including negative GBS screen 5 days prior to delivery. The mother received a complete antenatal steroid course with two doses of betamethasone intramuscular given 24 hours apart 3 days prior to delivery. She was started on latency antibiotics with ampicillin intravenous and azithromycin oral and was on treatment for 47 hours prior to delivery. Due to preterm labor at 23 weeks, she was given magnesium for neuroprotection 2 days prior to delivery. A male neonate of 23 and 4/7 weeks gestational age (GA) was delivered vaginally after prolonged rupture of membranes for 58 hours. The BW was $0.595 \mathrm{~kg}$ (58\%ile), and he was initially vigorous but required intubation and surfactant for respiratory distress with APGAR score $5,6,8$, at 1,5 , and 10 minutes, respectively. The neonate was admitted to the neonatal intensive care unit (NICU), placed on high-frequency oscillator for respiratory support due to high oxygen needs, started caffeine intravenous for apnea of prematurity, umbilical catheters inserted, and an evaluation for bacterial infection was initiated. The patient was initiated on empiric therapy of ampicillin and gentamicin, which was discontinued at 48 hours with negative blood culture. He was made nil per os with nutritional support via total parenteral nutrition. At 22 hours of life, trialed on conventional mechanical ventilation but failed at 60 hours of life due to increased oxygen needs and respiratory acidosis. The neonate was then transferred to our facility on day of life (DOL) 3 for further care in our specialized extremely low birth weight (ELBW) program. ${ }^{10}$

Following admission to our NICU, leukocytosis with white blood cells (WBC) of $19.8 \mathrm{~K} / \mu \mathrm{L}$ and bandemia of $15 \%$ were noted on DOL 4 and hour of life 99, prompting a septic workup with blood culture obtained from the umbilical arterial catheter. The umbilical venous catheter (UVC) was unable to draw to obtain a blood culture specimen. Empiric treatment with ampicillin and cefepime was initiated to avoid nephrotoxic medications and due to the nationwide shortage of cefotaxime. Later that day, his abdomen was discolored with an abdominal radiograph revealing a gasless abdomen in the absence of pneumoperitoneum or portal venous gas. The blood culture obtained from the umbilical arterial catheter was positive for gram negative rods in 9.6 hours, speciated as M. morganii, and the line was removed. Full sepsis workup was done on DOL 5 with repeat blood cultures, both peripheral and UVC, lumbar puncture, and a nonsterile urine culture due to inability to obtain catheterized specimen. Then antibiotic coverage broadened to meropenem and ampicillin as speciation of gram-negative

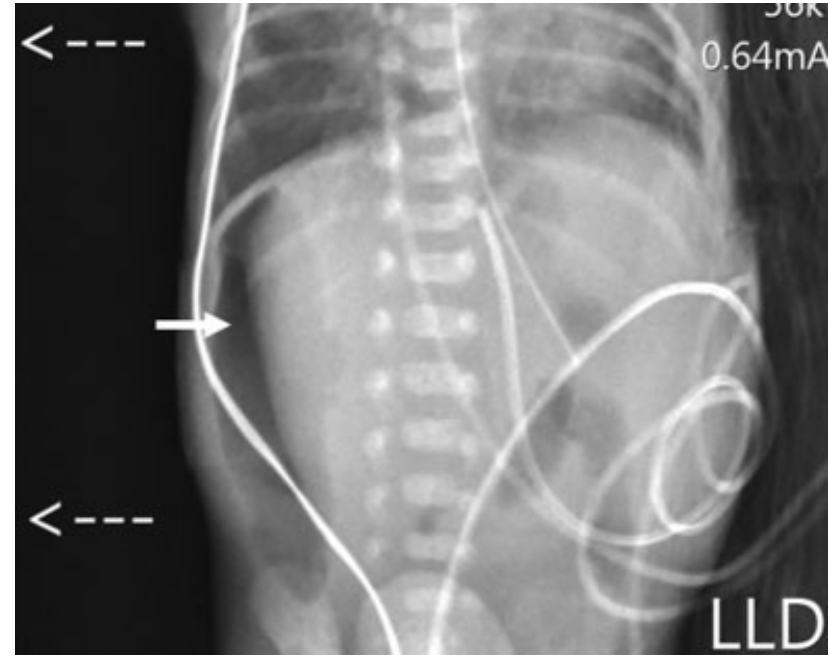

Fig. 1 Left lateral decubitus (LLD) showing pneumoperitoneum. <--indicates left side up. -> indicates free air.

rods was pending and to cover for extended spectrum $\beta$ lactamases. The cerebrospinal fluid (CSF) analysis showed 7 red blood cells per $\mu \mathrm{L}, 1 \mathrm{WBC}$ per $\mathrm{mm}^{3}, 206 \mathrm{mg} / \mathrm{dL}$ protein, $78 \mathrm{mg} / \mathrm{dL}$ glucose, and the CSF culture grew Enterococcus faecalis (pan-sensitive) that was initially thought to be a contaminate, being a different organism than the blood culture and with the infant clinically improving. The nonsterile urine culture was positive for multiple organisms: Staphylococcus epidermis, M. morganii, and E. faecalis, which were all felt to be reflective of GI flora rather than true infection. The peripheral culture returned positive for $M$. morganii but the UVC culture remained negative. After initial improvement on meropenem and ampicillin, on DOL 8, the infant clinically worsened with bilious emesis and increased abdominal discoloration. The infant had never been on enteral feeds, only oral care with colostrum starting on DOL 1 . An abdominal radiograph demonstrated free intraperitoneal air without pneumatosis (-Fig. 1), consistent with spontaneous intestinal perforation(SIP). The pediatric surgical team placed a peritoneal drain and the intraoperative peritoneal culture grew $M$. morganii. Antibiotic coverage was changed to include vancomycin and fluconazole given SIP. Once $M$. morganii sensitivities returned, in consultation with infectious disease specialists, meropenem was changed back to ampicillin and cefepime to complete an antibiotic course of 14 days from the SIP.

Given the association of M. morganii with EOS and culture positive at DOL 4 , the delivery record was revisited. The placental maternal culture grew E. coli, Streptococcus viridans, coagulase negative Staphylococcus, Enterococcus, Bacillus fragilis with placental fetal culture with same organisms in addition to Prevotella species. We theorize that our patient's $M$. morganii septicemia was, in fact, an early sign of bacterial translocation from the GI tract, which was related to the later development of SIP.

The infant continued to improve clinically and the rest of the infant's hospital course was relatively uncomplicated for his ELBW status. After healing of the intestinal perforation 


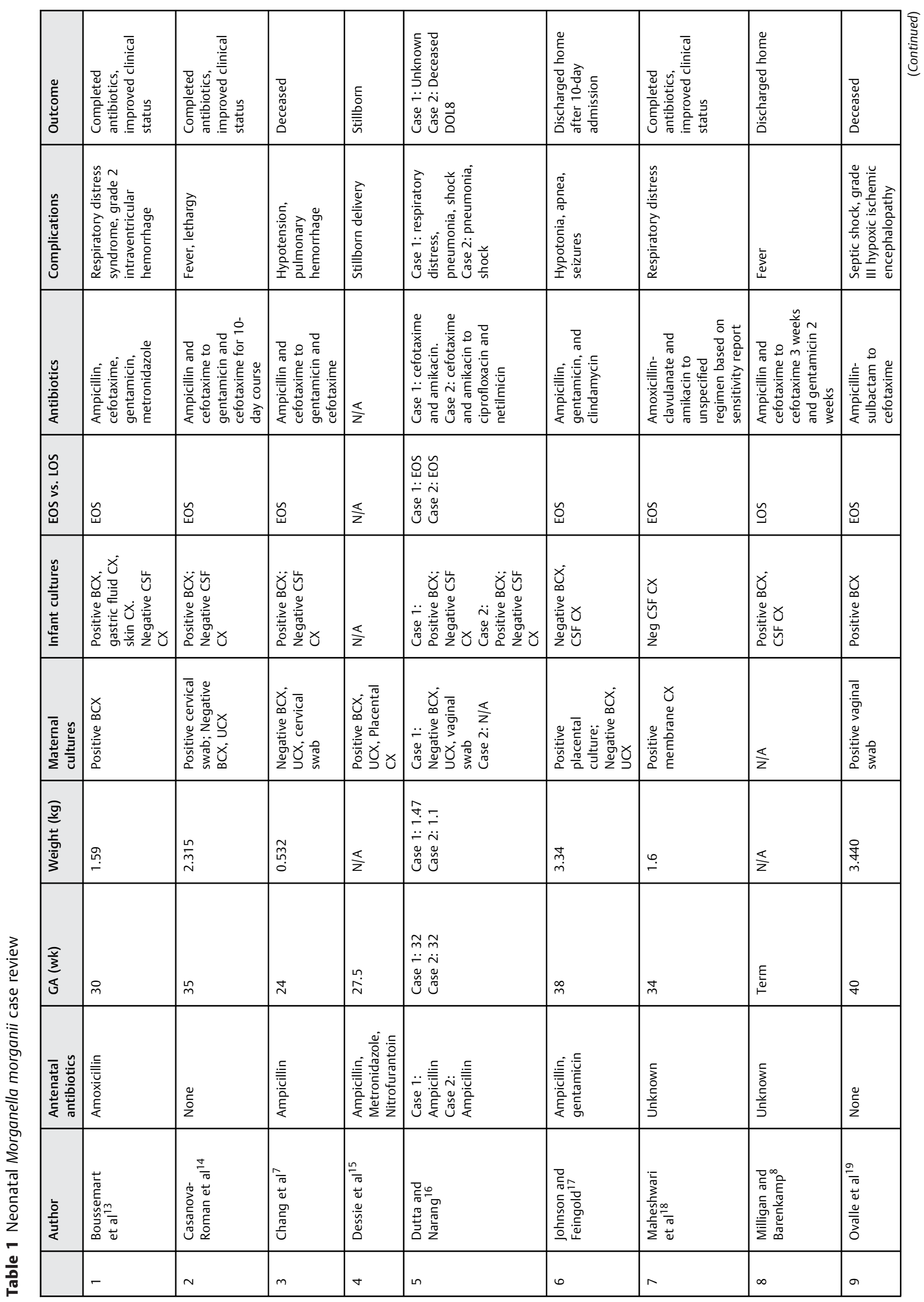




\begin{tabular}{|c|c|c|c|c|c|}
\hline 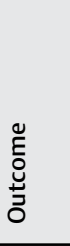 & 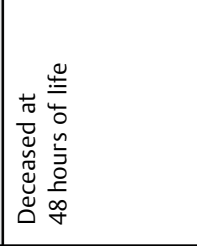 & 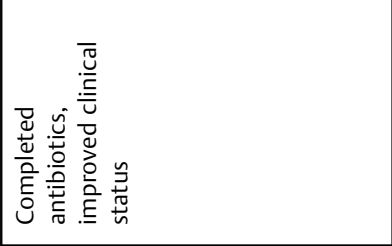 & 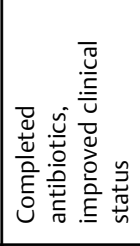 & 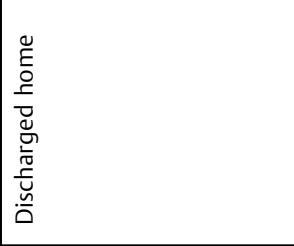 & 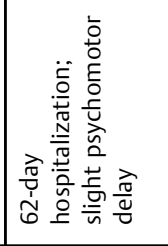 \\
\hline 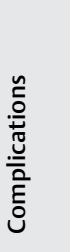 & 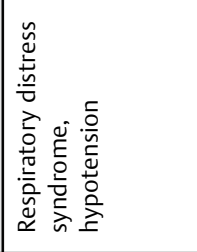 & 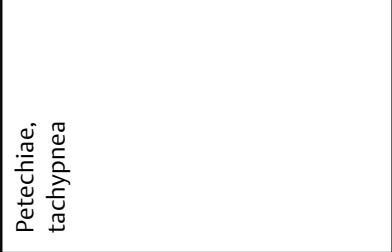 & 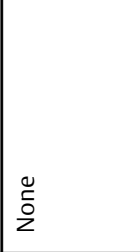 & 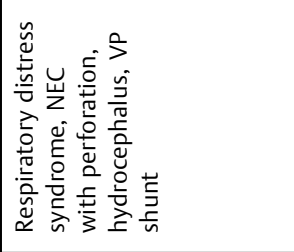 & 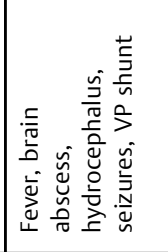 \\
\hline 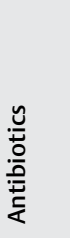 & 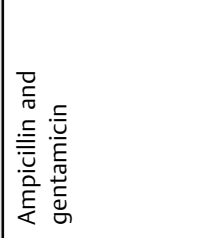 & 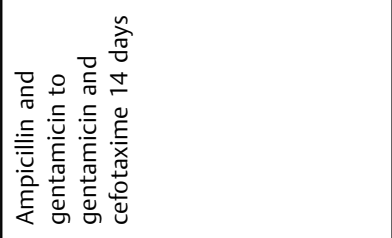 & 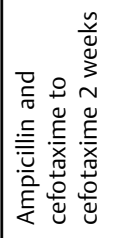 & 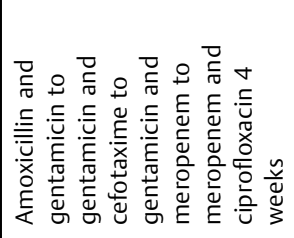 & 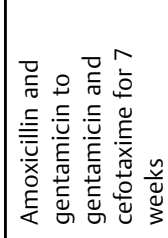 \\
\hline 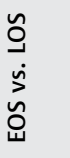 & 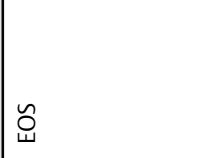 & 㟔 & $\tilde{0}$ & 号 & $\tilde{\varrho}$ \\
\hline 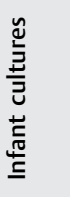 & 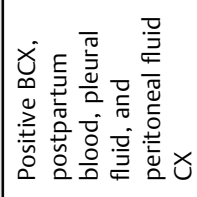 & 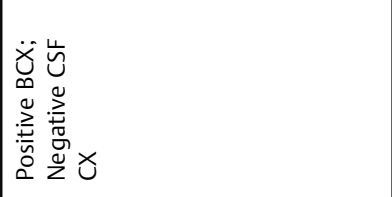 & 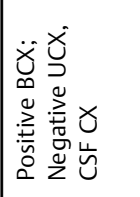 & 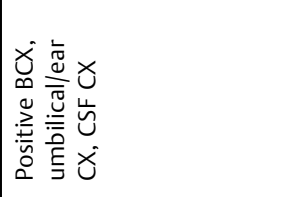 & 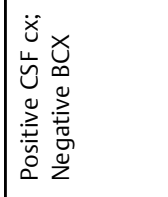 \\
\hline 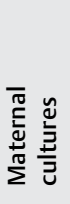 & 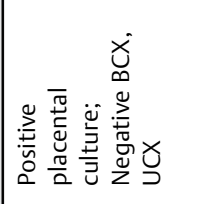 & 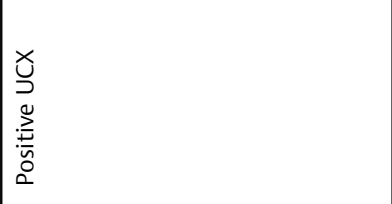 & 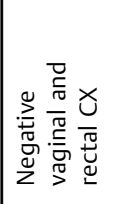 & 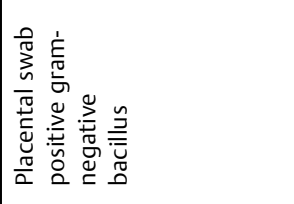 & $\frac{\ll}{z}$ \\
\hline 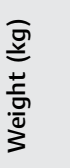 & $\begin{array}{l}\hat{n} \\
6 \\
0 \\
0\end{array}$ & $\frac{\infty}{i}$ & $\stackrel{\stackrel{n}{n}}{m}$ & $\begin{array}{l}\text { 命 } \\
\infty \\
0\end{array}$ & $\frac{\pi}{z}$ \\
\hline$\frac{\widehat{Y}}{3}$ & $\stackrel{ \pm}{\sim}$ & $\stackrel{\stackrel{n}{m}}{\text { r }}$ & 웅 & $\stackrel{\mathscr{N}}{\sim}$ & $\frac{\mathbb{z}}{z}$ \\
\hline 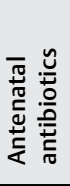 & 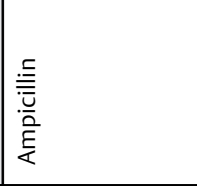 & 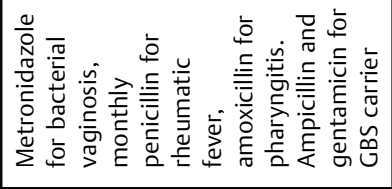 & $\frac{\ll}{z}$ & 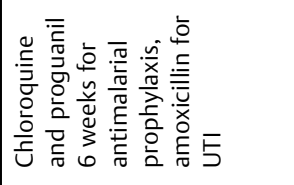 & $\frac{\mathbb{z}}{z}$ \\
\hline $\begin{array}{l}\text { 高 } \\
\frac{⿱ 亠 䒑}{2}\end{array}$ & 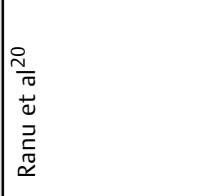 & 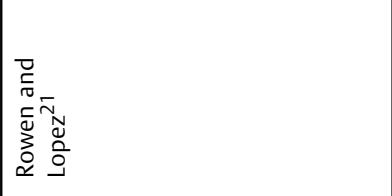 & 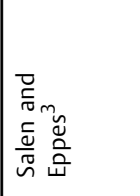 & 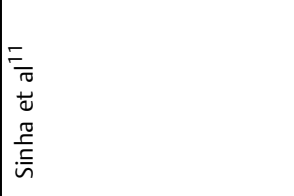 & 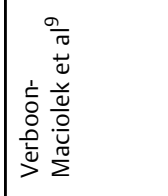 \\
\hline & $\circ$ & $=$ & $\simeq$ & $\stackrel{m}{\sim}$ & 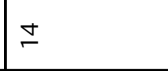 \\
\hline
\end{tabular}


and peritoneal drain removal, enteral feeds were slowly advanced and he tolerated full enteral feeds for the duration of his hospitalization. He received dexamethasone for evolving chronic lung disease and was extubated by DOL 30 , and weaned to room temperature by 37 and 4/7 weeks GA. His patent ductus arteriosus was treated with acetaminophen. The infant sustained bilateral germinal matrix hemorrhages with mildly dilated ventricles, which normalized by discharge. At corrected full-term cranial ultrasound, a small left-sided periventricular cyst was demonstrated; otherwise, no ventricular dilation or periventricular white matter changes identified. He developed stage 2 retinopathy of prematurity in both eyes, which did not require treatment. He was discharged home with his parents on DOL 126 at corrected GA of 41 and $4 / 7$ weeks.

He was seen in the high-risk infant follow-up clinic at corrected GA of 6 months. The patient was receiving feeding therapy, infant development services, and physical therapy. He was normal for age in cognitive skills, expressive language, fine motor skills, gross motor skills, and sensory processing on developmental assessment. He showed mild delays in receptive language and social emotional skills. An outpatient magnetic resonance imaging of the brain showed no significant structural or signal abnormality.

\section{Discussion}

M. morganii has emerged as a potential organism causing neonatal sepsis. A literature review of neonatal M. morganii infection shows few case reports causing variable clinical presentation (-Table 1). $)^{3,7-9,11-21}$ Most cases involve preterm infants and are associated with EOS. $M$. morganii is known to express AmpC $\beta$-lactamase that confers resistance to $\beta$-lactam antibiotics. ${ }^{11}$ It is theorized that antenatal maternal antimicrobial therapy with ampicillin precipitates the resistance. Of the nine cases that documented maternal antibiotic exposure, all received a $\beta$-lactam antibiotic ( - Table 1 ). In our case, the patient's mother also received ampicillin prior to delivery and his M. morganii showed ampicillin resistance. However, due to the onset of symptoms, his infection was considered LOS. He was initially on ampicillin and gentamicin, which were the standard for neonatal sepsis at the outside hospital, of which he completed a 48-hour course. With his elevated inflammatory markers on a screening complete blood count on DOL 5, he was started on empiric ampicillin and cefepime for neonatal sepsis with the goal to avoid nephrotoxic medications per EBLW protocol, with the choice of cefepime influenced by a nation-wide shortage of cefotaxime. The patient's culture susceptibilities demonstrated sensitivity to cefepime that was an effective choice for typical gram-positive and gram-negative organisms causing neonatal sepsis. Because of this inducible resistance, initial antibiotic therapy with ampicillin and gentamicin, which is standard in many units for neonatal sepsis, may provide inadequate coverage. Thus, a thirdgeneration cephalosporin and aminoglycoside have been suggested to provide more effective coverage.
Sources of M. morganii bacteremia have been previously studied with 20 to $22 \%$ of cases due to hepatobiliary tract infection. ${ }^{6,12}$ Other common sources causing similar rates of bacteremia included soft tissue infection and urinary tract infections. In our patient's case, we attribute the possible source of bacteremia from GI translocation, which may have contributed to his SIP. On the same day of the positive culture, DOL 4, we did note abdominal discoloration and a gasless abdomen on imaging. While his urine culture also demonstrated growth of M. morganii, it was a nonsterile specimen with polymicrobial growth that more likely represented GI flora. Of the cases found in our literature review, no other neonates with M. morganii LOS had intraabdominal complications, although one with EOS did have necrotizing enterocolitis. We describe the first case of a neonate with $M$. morganii bacteremia and SIP.

In adult literature analyzing bacteremia related to this organism, inadequate antibiotic coverage has been seen as a risk factor for increased mortality. However, of the few cases demonstrated in the neonatal literature, most of the cases of $M$. morganii infection in infants of similar GA had high mortality with death in 4 out of the 14 cases with available outcomes, or $28 \%$ mortality, and high morbidity despite appropriate antimicrobial coverage ( - Table $\mathbf{1}$ ). Preterm and low BW infants were more severely affected, with only one of the four extremely premature infants ( $<28$ weeks gestation) surviving from EOS. Of the LOS cases, none were extremely preterm, and all three survived. Despite the complication of SIP with the late onset M. morganii sepsis our infant had, he did survive and has been doing well developmentally with only mild delays.

\section{Conflict of Interest}

None declared.

\section{References}

1 Shane AL, Sánchez PJ, Stoll BJ. Neonatal sepsis. Lancet 2017;390 (10104):1770-1780

2 Stoll BJ, Hansen NI, Sánchez PJ, et al; Eunice Kennedy Shriver National Institute of Child Health and Human Development Neonatal Research Network. Early onset neonatal sepsis: the burden of group B Streptococcal and E. coli disease continues. Pediatrics 2011;127(05):817-826

3 Salen PN, Eppes S. Morganella morganii: a newly reported, rare cause of neonatal sepsis. Acad Emerg Med 1997;4(07):711-714

4 Stoll BJ, Hansen N, Fanaroff AA, et al. Late-onset sepsis in very low birth weight neonates: the experience of the NICHD Neonatal Research Network. Pediatrics 2002;110(2 Pt 1):285-291

5 Erlanger D, Assous MV, Wiener-Well Y, Yinnon AM, Ben-Chetrit E. Clinical manifestations, risk factors and prognosis of patients with Morganella morganii sepsis. J Microbiol Immunol Infect 2019;52(03):443-448

6 Liu H, Zhu J, Hu Q, Rao X. Morganella morganii, a non-negligent opportunistic pathogen. Int J Infect Dis 2016;50:10-17

7 Chang HY, Wang SM, Chiu NC, Chung HY, Wang HK. Neonatal Morganella morganii sepsis: a case report and review of the literature. Pediatr Int 2011;53(01):121-123

8 Milligan KL, Barenkamp SJ. Neonatal meningitis due to Morganella morganii. Clin Pediatr (Phila) 2013;52(05):462-464

9 Verboon-Maciolek M, Vandertop WP, Peters AC, Roord JJ, Geelen SP. Neonatal brain abscess caused by Morganella morganii. Clin Infect Dis 1995;20(02):471 
10 Morris M, Cleary JP, Soliman A. Small baby unit improves quality and outcomes in extremely low birth weight infants. Pediatrics 2015;136(04):e1007-e1015

11 Sinha AK, Kempley ST, Price E, Sharma BK, Livermore DM. Early onset Morganella morganii sepsis in a newborn infant with emergence of cephalosporin resistance caused by depression of AMPC beta-lactamase production. Pediatr Infect Dis J 2006;25(04):376-377

12 Lee I-K, Liu JW. Clinical characteristics and risk factors for mortality in Morganella morganii bacteremia. J Microbiol Immunol Infect 2006;39(04):328-334

13 Boussemart T, Piet-Duroux S, Manouana M, Azi M, Perez JM, PortLis M. [Morganella morganii and early-onset neonatal infection]. Arch Pediatr 2004;11(01):37-39

14 Casanova-Román M, Sanchez-Porto A, Casanova-Bellido M. Earlyonset neonatal sepsis caused by vertical transmission of Morganella morganii. Scand J Infect Dis 2002;34(07):534-535

15 Dessie SA, Dobariya V, Singh D, He P. A case of Morganella morganii-induced fetal demise. Cureus 2020;12(03):e7169
16 Dutta S, Narang A. Early onset neonatal sepsis due to Morganella morganii. Indian Pediatr 2004;41(11):1155-1157

17 Johnson JR Jr, Feingold M. Case of chorioamnionitis in an immunocompetent woman caused by Morganella morganii. J Matern Fetal Med 1998;7(01):13-14

18 Maheshwari A, Dutta S, Kumar P, Narang A. Early onset mixed Morganella and Klebsiella sepsis in a neonate. Indian J Pediatr 2001;68(07):671-672

19 Ovalle A, Martínez MA, Kakarieka E, García M, Salinas A. Sepsis neonatal precoz causada por transmisión vertical de Morganella morganii, en un embarazo de término [Fatal neonatal sepsis caused by vertical transmission of Morganella morganii. Report of one case.]. Rev Med Chil 2009;137(09):1201-1204

20 Ranu SS, Valencia GB, Piecuch S. Fatal early onset infection in an extremely low birth weight infant due to Morganella morganii. J Perinatol 1999;19(07):533-535

21 Rowen JL, Lopez SM. Morganella morganii early onset sepsis. Pediatr Infect Dis J 1998;17(12):1176-1177 